\title{
Dermal secretion physiology in Metastriata ticks; thermoregulation in the lone star tick Amblyomma americanum
}

\section{Paulina Maldonado-Ruiz}

Kansas State University

Brianna N. Davis

Kansas State University

Jessica J. Park

Kansas State University

Yoonseong Park ( $\square$ ypark@ksu.edu )

Kansas State University

\section{Research Article}

Keywords: Dermal glands, evaporative cooling, serotonin, $\mathrm{Na}$ /K-ATPase, Ixodidae, Rhipicephalus

Posted Date: February 16th, 2021

DOl: https://doi.org/10.21203/rs.3.rs-199579/v1

License: (c) (i) This work is licensed under a Creative Commons Attribution 4.0 International License.

Read Full License 


\section{Abstract}

Ticks are blood feeding ectoparasites that transmit a wide range of pathogens. The lone star tick, Amblyomma americanum, is one of the most widely distributed ticks in the Midwest and Eastern United States. Lone star ticks, like most three-host ixodid ticks, can survive in harsh environments for extended periods without consuming a blood meal. Physiological mechanisms that allow them to survive during hot and dry season include thermal tolerance and water homeostasis. Large quantity of dermal fluid secretions induced by mechanical stimulation of tick legs has been described in metastriate ticks including Amblyomma. We hypothesize that a function of tick dermal secretion is similar to the sweating in large homeothermal animals. In this study, we found that a contact with a heat probe at $45^{\circ} \mathrm{C}$ can trigger dermal secretion. We demonstrated that dermal secretion plays a role in evaporative cooling when ticks are exposed to high temperature. We observed that direct contact to a heat probe for 5 seconds at $\sim$ $52^{\circ} \mathrm{C}$ caused an exhaustive dermal secretion with $\sim 4 \%$ loss of body weight and resulted in the lethality in 24-hour, indicating that the secretion is associated with significant costs of water loss. We identified type II dermal glands having paired two cells forming large glandular structures. The secretion is triggered by an injection of serotonin and the serotonin-mediated secretion was suppressed by a pretreatment of Ouabain, a $\mathrm{Na}$ /K-ATPase blocker, implying that the secretion is controlled by serotonin and the downstream $\mathrm{Na}$ /K-ATPase.

\section{Introduction}

Ticks are blood feeding arthropods and the number one cause of vector-borne illness in the United States 1,2. The Family Ixodidae, hard tick containing the major vector species, is mainly divided into two groups; Metastriata group including Amblyomma, Rhipicephalus, and Dermacentor, and Prostriata group including Ixodes. ${ }^{2-4}$. The lone star tick, Amblyomma americanum, is a hard tick that belongs to the Metastriata group, which is widely distributed in the Midwest region of the United States ${ }^{5}$. This tick vectors pathogens, including Fransicella tularensis, Ehrlichia chaffeensis, and E. ewingii. Each are causative agents of Ehrlichiosis and a number of viruse ${ }^{6-8}$. This tick can also cause southern tick associated rash illness (STARI) and red meat allergy, which is caused by a specific glycan (alpha-gal or galactose-a-1,3-galactose) present in their salivary glands 9,10.

Multi-host hard ticks spend a relatively small part of their lifetime on the host for feeding. Over $90 \%$ of their lifetime is spent off-host, mostly in vegetated ground. Tick survival and success largely depends on their ability to maintain water balance during off-host periods in high temperature and low relative humidity $(\mathrm{RH}){ }^{11,12}$. Water absorption physiology in hard tick during the off-host periods has been reviewed previously ${ }^{13-15}$. Ticks capture water molecules in environmental vapor by using a hygroscopic saliva ${ }^{16}$ that is rich in chloride, potassium, and sodium ${ }^{11,17}$. Water absorption then takes place through the type I salivary gland acini ${ }^{18}$. In addition, active drinking directly from water drops has also been observed ${ }^{13,18,19}$. The physiology for obtaining water is to recuperate from the natural imminent water losses that occur through evaporation and excretion ${ }^{3,20-22}$. 
Ticks have thick sclerotized cuticle covered with wax ${ }^{13}$ preventing evaporative water loss. However, an important route of water loss through the integument may be secretion through dermal glands which accounts for $2.3-2.5 \%$ of the body weight in the case of $R$. sanguineus ${ }^{23}$ and $4 \%$ in $A$. americanum (in this study). In metastriate ticks, the dermal secretion occurs through a large number of a subset of dermal glands opened to dorsal and ventral surface, which has been demonstrated to be triggered through mechanical stimulation ${ }^{3,24}$. It has been proposed that the primary function of this secretion is to defend against predators and pathogens as it contains squalene and other unknown toxic compounds ${ }^{25-27}$. Other reports also show that the gland secretion contains compounds with pheromonal activity for aggregation, such as: $o$-nitrophenol and methy-salicylate in the fed male $A$. variegatum ${ }^{28}$ and in $A$. haebreum ${ }^{29}$. In addition, it has been also reported that dermal excretion through mechanical stimulation provides heat tolerance in $R$. sanguineus ${ }^{30}$, opening possibilities of multiple functions of the dermal secretion, although the analytical studies for different types of secretion is yet lacking. We hypothesize that the tick dermal secretion, large volume of fluid containing small portion of the active compounds, functions for evaporative cooling like the case of sweating in large animals.

In this study, we propose that dermal secretion allows ticks to cope with a sudden exposure to hot temperature by using evaporative cooling of the body. We show here heat-probe induced dermal secretions and rapid evaporative cooling, supporting the hypothesis. The dermal secretion physiology is further investigated by identifying the molecular components, serotonin and $\mathrm{Na}$ /K-ATPase, involved in the control of the dermal secretion.

\section{Results}

\section{Tick dermal secretion is induced by a contact to the heat probe}

The dermal secretion observed for the dorsal side was induced by a dorsal contact of the thermal probe (Fig. 1 and supplementary Video S1) at $35^{\circ} \mathrm{C}$ in $12.5 \%$ individuals, and $100 \%$ individuals responded at 45 ${ }^{\circ} \mathrm{C}$ (Fig. 2a) in A. americanum. When the treatments were made by sequential increases in the probe temperature on the same individual, where rapid acclimation to prior exposure to the heat probe could occur, the temperature required for induction of dermal secretion was $42{ }^{\circ} \mathrm{C}$ in $\mathrm{A}$. americanum, Other tick species also showed secretion responses with moderate levels of shifts in the sensitivities: $R$. sanguineus $\left(45^{\circ} \mathrm{C}\right.$ ) and $D$. variabilis $\left(50^{\circ} \mathrm{C}\right)$ where total percent response was 90 and $65 \%$ respectively (Fig. $\left.2 \mathrm{~b}\right)$. No dermal secretion was observed in Ixodes scapularis.

When $A$. americanum secretion was induced by the heat-probe at $55-58{ }^{\circ} \mathrm{C}$ for up to 5 seconds, the exhaustive dermal secretion resulted in significant loss of the weight, $4.1 \%$ loss from the average $6.1 \mathrm{mg}$ to $5.8 \mathrm{mg}(n=40)$. These group of the ticks were all dead in a day kept in $95 \% \mathrm{RH}$ after the treatment. The temperature in an edge of a typical tick habitat in Kansas in a sunny summer day with $33^{\circ} \mathrm{C}$ ambient temperature was found to be in a range of $\sim 55-65^{\circ} \mathrm{C}$ on the surface of soil and $\sim 37-50{ }^{\circ} \mathrm{C}$ on the surface of grasses (Fig. 2c). 
In an investigation of the effect of dermal secretion on evaporative cooling, the temperature change monitored on the dorsal surface showed significantly higher cooling rate in the ticks with dermal secretions than in those without secretion (Fig. 3a). When the cooling rate was regressed with an exponential decay formula, the slopes of decay were significantly different; -1.7 compared to that of no secretion -1.2 (Fig. 3b). The evaporative cooling in the ticks with the secretion resulted in 1.2 degrees cooler at the 10 second after the contact with heat probe (Fig. 3a).

Dermal secretion is induced by serotonin and inhibited by Ouabain

In injection of different biogenic amines, neuropeptides and secondary signaling molecules, we found that a biogenic amine serotonin triggered an immediate dermal secretion at $1 \mathrm{mM}$ (Table 1). This response was observed in $75 \%$ of $A$. americanum (Fig. 4 and supplementary Video S2). In other Metastriata ticks, we observed that $75 \%$ individuals responded in $R$. sanguineous, $50 \%$ in $D$. variabilis, whereas no response was observed in a Prostriata Ixodes scapularis (Fig. 4a). Dose-response of serotonin showed that the dose required for the secretion is higher than $100 \mathrm{mM}$ in $10 \mathrm{~nL}$ injection and reached to the maximum response to a plateau at $1 \mathrm{mM}$ with $75 \%$ responders, which was also found in an increased concentration to $10 \mathrm{mM}$ (Fig. 4b).

We expanded the study to investigate the downstream machinery for fluid transport. A pre-treatment of Ouabain, a Na/K-ATPase inhibitor, by an injection of $10 \mathrm{~nL} 100 \mathrm{mM}$ at the $-30 \mathrm{~min}$. of the serotonin injection, blocked the secretion response to the serotonin injection. Ouabain significantly lower the response to serotonin than that in control (water injection) ( $p=0.0134$ in a Chi-square test) (Fig. 4c).

An interesting observation worth to be mentioned in this set of experiment was bilateral asymmetric responses (Supplementary Table S1). In one batch of ticks, when ticks were injected on the left side, majority of the ticks responded with the secretion on the right side and vice versa. However, in another batch of ticks, the secretion responses were observed on the same side of injections; right side injections resulted in the right side response and vice versa. (Table S1).

Dermal secretion and type II dermal glands were visible with the aid of blue fluorescent light

After the induction of dermal secretion by a heat-probe to the legs, we found that the drops of dermal secretions, $\sim 2 \mathrm{~nL}$ from each dermal pore, which were dried immediately on the surface of the integument, had blue fluorescence under CFP filter (Fig. 5a-d) in a fluorescence stereoscope. Dissections and visualization of the dermal glands were performed after we blocked the secretory response by an injection of local anesthetic Procaine hydrochloride to naïve ticks at 10 minutes prior dissection $(n=13)$ to preserve the intact gland structure during the dissection procedure, which induces the secretion and collapses the gland. The Procaine injected individuals were tested by a heat-probe before dissection to ensure lack of secretion as the anesthetic response. Only the individuals with a negative secretory response $(9 / 13)$ were used for dissection and visualization for the internal structure. The glands under the fluorescent light with CFP filter set showed blue fluorescent glandular structures in both dermal and ventral layers (Fig. 5e-g). The blue fluorescent glands were rich in the festoon region, while a number of 
the glands were also observed in the region surrounding the base of coxa and also in the regions near the clusters of dorso-ventral muscles.

Confocal images of dorsal and ventral integuments showed the same patterns of localization for the type II gland structures as were observed in the CFP filter set; the type II glands mainly in the tick festoons and also in the area surrounding the coxal regions and near the dorso-ventral muscles, while the glands lost the intactness of the round shapes during the sample processing. A pair of large nuclei associated with balloon-like thin membrane structure was observed on the internal surface of epidermal cell layer. All the staining reagents that we employed, Na/K-ATPase, cell membrane (CellMask ${ }^{\mathrm{TM}}$ ), betatubulin, and HRP (neural marker in insects), helped the visualization of the type II dermal glands. The antiHRP antibody and CellMask ${ }^{\mathrm{TM}}$ showed the robust membrane staining (Fig. 6a-f). Anti-beta-tubulin antibodies stained subcellular part of the glands, but with high variability among different glands (Fig. 6b-f). The entire membrane for small number of glands were stained for beta-tubulin (Fig. 6c, e, and f), but mostly having only small spots on the membrane (Fig. 6b), whereas all type I glands were positive for the beta-tubulin (Fig. 6d). Often, we observed clustered subcellular region of basal part of the glands that was positive for $\beta$-tubulin (Fig. $6 \mathrm{e}$ and $\mathrm{f}$ ). Anti-HRP stained the clusters of the cells in the type I glands (Fig. $6 \mathrm{~d}$ and $\mathrm{f}$ ) and the membrane of type II glands. Anti-Na/K-ATPase antibody stained small spots on the membrane of type II glands (Fig. 6h and j).

In microtome sections of the dermal layers, two types of dermal glands were obviously categorized: narrow duct with a cluster of small cells for type I (Fig. 7a-c) and the large dermal glands connected to the wide dermal pores for type II (Fig. 7d-f). The location and the shape of the type II glands are well correlated with the structures observed in fluorescence stereoscope and confocal images. Each wide dermal pore is mostly connected to a paired two glandular structures, which was shown in the confocal microscopy with two large nuclei

\section{Discussion}

We demonstrated that tick dermal secretion is triggered by contact with a high temperature substrate in this study. Evaporation of the dermal secretion significantly helped the rapid cooling of the body. These data support that a function of tick dermal secretion is to provide the evaporative cooling of the body in hot environments, which can be up to $65^{\circ} \mathrm{C}$ shown in a typical tick habitat in a summer day in Kansas (Fig. 2c).

Evaporative cooling in small invertebrate is an unexpected observation; while, endothermic/homeothermic large animals are well known for dermal secretion, sweating, for evaporative cooling in thermoregulation through apocrine and eccrine glands ${ }^{31}$. Small arthropods, having high surface to volume ratio, are vulnerable to evaporative loss of water. The integument covered with a wax layer in arthropods is efficiently preventing evaporative loss of water. The cost of dermal secretion is shown by the death of the individual having exhaustive dermal secretion by a contact of $55-58{ }^{\circ} \mathrm{C}$ heat probe for 5 seconds. Excessive loss of fluid ( $4.1 \%$ of body weight) is likely leading to death although the 
accurate cause of the death in this case needs to be further investigated. Despite of the costs of water loss, tick dermal secretion is likely important for tick survival with the tight control of the secretion. Indeed, previous studies have shown that the ticks with dermal secretion can survive better when they are exposed to high temperature. This finding was reported in $R$. sanguineus where dermal secretion provided heat tolerance after ticks were mechanically stimulated through leg pinching ${ }^{30}$. In this study, the tick survival after $1 \mathrm{hr}$. heat shock in naïve ticks was $27 \%$ at $52^{\circ} \mathrm{C}$ and $18 \%$ at $54^{\circ} \mathrm{C}$, whereas ticks that experienced dermal secretion by pinching the legs were much more tolerable in the same condition, i.e., $93 \%$ survival at $52^{\circ} \mathrm{C}$ and $89 \%$ at $54^{\circ} \mathrm{C}$. The authors suggest that heat tolerance may be the result of internal changes in the dermal glands that occur after secretion. The physiology behind this tolerance needs to be investigated further.

The secretion is proposed to contain defensive compounds against predators and pathogens. Such toxic compounds, like antimicrobials and squalene, can be externalized through pore openings on the cuticle surface ${ }^{25,26}$ and may act as allomones against predatory ants like the case of squalene ${ }^{23}$. An earlier study identified $o$-nitrophenol and methyl salicylate in the type II glands of fed male ticks of $A$. variegatum and $A$. hebraeum, which are thought to have aggregation pheromonal activities ${ }^{28,29}$. In this study, we add an additional function of the dermal secretion for thermoregulation by evaporative cooling.

Two types of dermal glands and pores have been described in $R$. sanguineus ${ }^{32}$, namely type I and type II. Type I glands are also known as small gland ${ }^{33}$ and type II are known as large wax gland or type A gland 33-35. Dermal secretion has been reported to occur presumably through type II dermal glands connected to sensilla sagittiformia types of pore, which are exclusive to metastriate ticks ${ }^{36}$ and absent in prostriate ticks ${ }^{34,37}$, although previous reports have not been able to directly link this type of secretion with the specific type II glands. A number of our observations support that the type II gland we described is the gland for the dermal secretion, i.e., the number, size, and location of the glands are equivalent to the external pore that produces secretions. The blue fluorescence we observed in the secretion is also localized to the type II glands.

Gland morphology consisted of paired two cells each having thin membrane forming a balloon-like sac, which is similar to the previous description for $R$. sanguineus ${ }^{28}$. Immunohistochemistry of the type II glands suggests the absence of neuronal projections connecting the dermal gland, based on the lack of axon-like projections in HRP-immunoreactivity. Interestingly, the HRP immunoreactivity, which presumes staining neuronal associated glycans in arthropods, also appears to be present in subcellular structures of the gland itself. Noticeably, however, the anatomically categorized dermal gland type II appear to be having subcategories based on the blue fluorescence of the contents and immunoreactivities in the dermal gland sac for $\mathrm{Na} / \mathrm{K}$-ATPase. The dermal secretion often contained non-fluorescent oily product externally (Fig. 5d), and the type II glands in the internal structure often was not fluorescent in the CFP filter set. Na/K-ATPase and beta-tubulin immunoreactivities were also vary for different type II glands (Fig. 6b, c, h, and j). It is not clear whether the variation is caused by the intrinsic, environmental, developmental variations at this time. 
While the multifunctional dermal secretion is a novel biological innovation, it costs a large amount of water loss as is shown by the lethality caused by exhaustive secretion. Therefore, the dermal secretion is likely a process tightly controlled by neural/hormonal mechanisms. In ticks, the water homeostasis is a tightly controlled physiology. The excretions occurring through the saliva and hindgut are controlled by neural and hormonal controllers ${ }^{21,38}$. An immediate response of dermal secretion upon the injection of serotonin (sub-second) favors serotonin as a direct hormonal/neural factor for activating the dermal glands. Blocking the serotonin-mediated dermal secretion by Ouabain suggests that the main downstream transporter is $\mathrm{Na} / \mathrm{K}-\mathrm{ATP}$ ase in the dermal glands. This is supported by our IHC results showing granular spots of $\mathrm{Na}$ /K-ATPase staining in both type I and type II glands (Fig. $6 \mathrm{~h}$ and j), So far, the roles of $\mathrm{Na} / \mathrm{K}$-ATPase in ticks have been described for the secretory activities in the salivary gland type 2 and 3 acini and resorptive activity in the salivary gland type I. A previous study for RNAi of $\mathrm{Na} / \mathrm{K}$ ATPase in a feeding stage tick found that the phenotype was associated with incomplete feeding and limited cuticle expansion during feeding ${ }^{39}$. An involvement of $\mathrm{Na} / \mathrm{K}$-ATPase in the dermal secretion suggests another function of $\mathrm{Na} / \mathrm{K}$-ATPase in different physiological processes.

In conclusion, tick dermal secretion provides evaporative cooling when the ticks encounter the contact with hot substrates in microhabitats. This could be a factor contributing to their extended survival rates and habitat expansions to geographical areas with hot temperature. There are two main molecular controllers of the dermal secretion pathway: serotonin and Na/K-ATPase. The dermal secretion involved in osmoregulatory physiology and thermoregulation may offer a vulnerable tick physiology that can be targeted in development of tick control measures. Current study brought in an interesting aspect of this exocrine gland that is responsible for the massive amount of dermal secretion in a short time.

\section{Materials And Methods}

$\underline{\text { Ticks }}$

Unfed 2 to 3-month old female adult $A$. americanum, $D$. variabilis, $R$. sanguineus, and $I$. scapularis were obtained from the Oklahoma State University tick rearing facility. They were kept at room temperature and $>95 \% \mathrm{RH}$ until used for experiments. Ticks were kept at $28{ }^{\circ} \mathrm{C}$ and $>90 \% \mathrm{RH}$ prior to experiments.

Induction of dermal secretion by heat probe

Ticks were immobilized on a flat surface with double-sided sticky tape (3M Scotch permanent mounting tape, MN, USA). The dorsal surface of the tick was exposed to heat by a direct contact with a heat probe made of 28-gauge nichrome 80 wire for 1-5 sec., depending on the experiments specified. The temperature of the wire was controlled by a current controller (Stoelting Co, IL, USA) and the temperature was monitored by a thermal camera (SeekTermal, CompactXR).

In order to measure the threshold temperature for secretion response in $A$. americanum, we treated the ticks with different temperatures for $1 \mathrm{sec}$. with the temperature increasing from 30 to $70{ }^{\circ} \mathrm{C}$ by $5^{\circ} \mathrm{C}$ 
interval. Eight ticks were tested at each temperature to avoid acclimation or sensitization of ticks to prior exposure to the heat probe. Another modified method was used for measuring the heat sensitivity in the test including other Metastriata ticks (A. americanum, $R$. sanguineus and D. variabilis). In this test, individual ticks were exposed to the probe repeatedly with sequential increments of the probe temperature. In each trial the probe temperature was sequentially increased by $\sim 3$ to $4^{\circ} \mathrm{C}$ ( $n=10$ for $R$. sanguineus, $n=12$ for $A$. americanum, and $n=12$ for $D$. variabilis). If the dermal secretion was not observed before 5 seconds of contact at the given temperature, the probe was detached from the tick and the next higher temperature was set for another contact that was followed by $\sim 1 \mathrm{~min}$. interval. A control group was treated with the probe at room temperature (RT) to ensure the response was not due to mechanical simulation by contact from the probe. During this operation, the thermal image was recorded and analyzed by SeekThermal application software (V2.1.9.1) and the image processing software (V2.6.1.12).

To assess the impact of dermal secretion on the body cooling rate, a set of 5 ticks per group were used; a group with no sweat and with sweat. Both were treated with the probe at the temperature between 40-42 ${ }^{\circ} \mathrm{C}$. Ticks reported as; no sweat means they did not exhibit the secretion after 5 seconds of the heat probe contact. Thermal images were analyzed as 20 frames per second using Image J $1.53 \mathrm{a}{ }^{40}$. The cooling rate was assessed by the average pixel values of 3 different regions of interest (ROI) surrounding the probe contact point on the tick surface. The average pixel value of the ROI was used for accurate estimation of temperature. The rate of lowering the temperature from $40^{\circ} \mathrm{C}$ in each tick were fitted to an ExpDec 1 curve (OriginPro 2020b, Fig 3b) and values obtained for each rate of decay, $A_{1} / t 1$, were used in a Student's T-test for the analysis (Fig $3 \mathrm{~b}$ ). ExpDec1 regression provided the fits with $\mathrm{R}$ values in the range of 0.97 to 0.99 . The regression and the data analyses were conducted in OriginPro 2020b (9.7.5.184).

To assess the amount of weight loss after exhaustive dermal secretion, the ticks kept in high $\mathrm{RH}$ (>95\%) for one day were weighted before and after the contact with heat probe at $55-58^{\circ} \mathrm{C}$ for $5 \mathrm{sec}$. The ticks after the treatments were placed on the high $\mathrm{RH}$ for recovery and monitored for survivorship.

\section{Pharmacology of dermal secretion}

To identify the neural or hormonal components involved in dermal excretion, we injected a series of biogenic amines, neuropeptides, and secondary signaling messengers. The chemicals used for biogenic amines were: Octopamine (( \pm )-Octopamine hydrochloride, Sigma, Cas\#00250), Norepinephrine (( \pm )-Norepinephrine (+)-bitartrate salt, Sigma, Cas\#3414-63-9), Dopamine (Dopamine hydrochloride, Sigma, Cas\#H8502), Serotonin (5-hydroxytriptamine hydrochloride, Sigma Cas\#H9523), for neuropeptides were: SIFamide (AYRKPPFNGSIFamide, ${ }^{41,42}$ ), MIP-1 (Mioinhibitory peptide-1, ASDWNRLSGMWamide, ${ }^{41,42}$ ), Proctolin (RYLPT, ELV-1 (Elevenin, LDCRKYPFYYRCRGISA, ${ }^{43}$ ), for secondary signaling messengers were: Dibutyryl cAMP (Dibutyryl adenosine 3',5'-cyclic monophosphate sodium salt, Santa Cruz Biotechnology, Cas\# 16980-89-5), Forskolin (Sigma, Cas \# F3917), Dibutyryl cGMP (Santa Cruz Biotechnology, Cas\# 51116-00-8) SNAP (N-(acetyloxy)-3-nitrosothiovaline, Cayman chemicals Cas\#67776-06-1). 
The compounds dissolved in water were injected through the base of the second coxal segment from lateral side using a Nanoject III nano-injector (Drummond Scientific Company, PA, USA) (Table 1). All injections were made for $10 \mathrm{~nL}$ unless it is specified. The strong secretion inducer, serotonin, was further tested for obtaining the full dose-responses.

We tested the role of $\mathrm{Na}^{+} / \mathrm{K}^{+}$-ATPase in the dermal secretion by a pretreatment of the tick with Oubaian, a $\mathrm{Na}^{+} / \mathrm{K}^{+}$-ATPase inhibitor. Ticks were injected with $10 \mathrm{nl}$ of $100 \mu \mathrm{M}$ Oubaian $30 \mathrm{~min}$ before injection of serotonin $(10 \mathrm{nl}$ of $1 \mathrm{mM}$ ). In rare cases ( 3 out of 20 ), the ticks that showed dermal secretion in Ouabain injection were excluded in data analysis because they were considered as the response to mechanical stimulation by insertion of the needle, which also occurred in water control.

\section{Localization of dermal secretion and the anatomy}

Naïve A.mericanum females were used to visualize the structure of the dermal gland on the dorsal and ventral dermal integuments. Immobilized ticks were placed on a double-sided sticky tape and treated by a heat contact to the legs. Dorsal dermal secretion was observed under fluorescent light with CFP filter set (excitation BP436/7, dichromatic mirror 455, and emission filter 470LP). The majority of dermal secretions displayed fluorescence drops with the CFP filter set. For internal view of the dermal glands, naïve ticks were injected with a local anesthetic, $20 \mu \mathrm{l}$ of $740 \mathrm{mM}$ of Procaine hydrochloride (SigmaAldrich, St. Louis, MO, USA), to prevent the dermal secretion triggered in the processes of dissection, which empties the gland contents. Ten minutes after procaine injection, the dorsal integument of ticks was removed with a surgical scalpel to visualize the internal glands. External and internal images were captured using a camera (DFC400) attached to a stereo microscope (M205FA; Leica, Heerbrugg, Switzerland) with the CFP filter set.

For confocal imaging of the glands, cellular structures, and molecular components, dorsally opened tissues were washed with PBST fixed in 4\% paraformaldehyde for 3 hours. The tissues were then incubated with 5\% normal goat serum (Jackson ImmunoResearch), containing the target antibodies overnight at room temperature. Immunohistochemistry $(\mathrm{IHC})$ was performed using beta-tubulin mouse antibody (GenScript, Piscataway, NJ, USA) at a final concentration of $0.5 \mathrm{mg} / \mathrm{ml}$, mouse monoclonal antibody (a5) raised against chicken $\mathrm{Na}$ /K-ATPase (Developmental Study Hybridoma Bank, University of lowa) at $4.4 \mu \mathrm{g} / \mathrm{ml}$. To localize $\mathrm{Na} / \mathrm{K}$-ATPase, we used a procedure already established by our laboratory 44. Following primary antibody incubation, tissues were washed with PBST and subsequently incubated overnight at room temperature with the secondary antibody, goat-anti-mouse IgG antibody conjugated with Alexa Fluor 488 (Molecular Probes, Eugene, OR, USA). In addition, goat polyclonal antibody against horseradish peroxidase (HRP) conjugated with Cyanine Cy ${ }^{\mathrm{Tm}} 3(8 \mu \mathrm{g} / \mathrm{ml}$, Jackson Immunoresearch, West Grove, PA. USA), containing $5 \%$ NGS, was used. The HRP-antibody has been used for characterization of the tissues having neural properties in insect ${ }^{45}$, due to its immunoreactivity against an N-linked oligosaccharide epitope expressed on neuronal glycoproteins in insects ${ }^{46}$. After the secondary antibody incubations, tissues were washed with PBST, incubated in 300nM 4',6'-diamino-2-phenylindole (DAPI, Sigma) or $2.5 \mu \mathrm{g} / \mathrm{ml}$ Hoechst 33342 (Invitrogen, Carlsbad, CA, US) and 40 fold dilution of Phalloidin 
conjugated with Alexa Fluor ${ }^{\text {TM }} 555$ (used for actin staining), (Molecular Probes, Eugene, OR, USA) or 5 $\mu \mathrm{g} / \mathrm{ml}$ CellMask $^{\mathrm{TM}}$ (Invitrogen, Carlsbad, CA, US) for 10 minutes, washed for 30 minutes and then mounted in glycerol. Images were captured with a confocal microscope (Zeiss LSM 700).

In microtome sections to visualize the cuticle integument and epidermal layers, ticks were cut into 2-3 pieces directly alive or after snap-freezing using liquid Nitrogen. Ticks were fixed for 3 hours at room temperature in non-alcoholic Bouin's fixative and washed with PBS containing $0.5 \%$ Triton X-100 (PBST). Samples were dehydrated with series of increasing ethanol solution (50 to 95\%) and an additional cuticle plasticization step was conducted by placing samples in n-Butanol ( 3 hours incubation at room temperature with rotation). Following dehydration, samples were placed in $100 \%$ chloroform for overnight at $60{ }^{\circ} \mathrm{C}$ and transferred to paraffin for up to $96 \mathrm{hrs}$. Tissue sections were made by using a Leica microtome at 8 to $10 \mu \mathrm{m}$ thickness and placed on a slide with $0.5 \%$ gelatin. Samples were dried in an incubator at $40{ }^{\circ} \mathrm{C}$ overnight. Deparaffinization was conducted using xylene, tissues were rehydrated with decreasing series of ethanol solutions (95 to 50\%) and washed with PBST.

For visualization of dermal layers under bright field, staining with methylene blue (10 seconds) was conducted, using solution II from the Hema $3^{\mathrm{TM}}$ staining kit (Protocol ${ }^{\mathrm{TM}}$, Fisher Scientific, Waltham, MA, USA). Slides were visualized using a Nikon Eclipse E800 compound microscope.

\section{Data availability}

Data will be deposited in the public repository if the manuscript is accepted for publication.

\section{Declarations}

\section{Acknowledgments}

This study is contribution no. 20-138-J from the Kansas Agricultural Experiment Station. This research was partially funded by Department of Defense TBDRP, D01 W81XWH-18-1-0255 and National Institute of Health; Grant Number: R21Al135457.

\section{Author contributions}

LPMR conducted the experiments, analyzed data, wrote the manuscript; BD and JJP conducted heating and injection experiments; YP funded the study, designed the experiments, wrote the manuscript and analyzed data.

\section{Declaration of interests}

The authors declare no competing interests. 


\section{References}

1. CDC. Tickborne diseases in the United States: A reference manual for healthcare providers. (2018).

2. Mullen, G. R. \& Durden, L. A. in Medical and veterinary entomology 517-558 (Academic Press, Amsterdam ; 2002).

3. Sonenshine, D. E. \& Roe, R. M. Biology of Ticks Volume 1. (Oxford University Press, Incorporated, 2013).

4. Sonenshine, D. E. \& Roe, R. M. Biology of Ticks Volume 2. (Oxford University Press, Incorporated, 2013).

5. CDC. Map of established Amblyomma americanum tick populations in the United States 2019. (2019).

6. Mixson, T. R. et al. Prevalence of Ehrlichia, Borrelia, and Rickettsial agents in Amblyomma americanum (Acari: Ixodidae) collected from nine states. J Med Entomol 43, 1261-1268, doi:10.1603/0022-2585(2006)43[1261:poebar]2.0.co;2 (2006).

7. Sayler, K. A. et al. Prevalence of tick-borne pathogens in host-seeking Amblyomma americanum (Acari: Ixodidae) and Odocoileus virginianus (Artiodactyla: Cervidae) in Florida. J Med Entomol 53, 949-956, doi:10.1093/jme/tjw054 (2016).

8. Tokarz, R. et al. Identification of novel viruses in Amblyomma americanum, Dermacentor variabilis, and Ixodes scapularis ticks. mSphere 3, e00614-00617, doi:10.1128/mSphere.00614-17 (2018).

9. Crispell, G. et al. Discovery of alpha-gal-containing antigens in North American tick species believed to Induce red meat allergy. Front. Immunol. 10, 1-16, doi:10.3389/fimmu.2019.01056 (2019).

10. Park, Y. et al. Alpha-Gal and cross-reactive carbohydrate determinants in the N-glycans of salivary glands in the lone star tick, Amblyomma americanum. LID - 10.3390/vaccines8010018 [doi] LID - 18. Vaccines (2019).

11. Needham, G. R. \& Teel, P. D. in Morphology, physiology and behavioral biology of ticks (ed J.R. Sauer and J.A. Hair) Ch. 5, 100-151 (Ellis horwood limited, 1986).

12. Norval, R. A. Studies on the ecology of the tick Amblyomma hebraeum Koch in the eastern Cape province of South Africa. II. Survival and development. J Parasito/ 63, $740-747$ (1977).

13. Sonenshine, D. E. in In Biology of Ticks Vol. 1 (ed D.E. Sonenshine and R. M. Roe) Ch. 22, 398-412 (1991).

14. Sauer, J. R. \& Hair, J. A. Water balance in the lone star tick (Acarina: Ixodidae): the effects of relative humidity and temperature on weight changes and total water content. $J$ Med Entomo/ 8, 479-485, doi:10.1093/jmedent/8.5.479 (1971).

15. Wharton G.W. , a. R. A. G. Water vapor exchange kinetics in insects and acarines. Annual Review of Entomology 23, 309-328, doi:10.1146/annurev.en.23.010178.001521 (1978).

16. Rudolph, D. \& Knulle, W. Site and mechanism of water vapour uptake from the atmosphere in ixodid ticks. Nature 249, 84-85, doi:10.1038/249084a0 (1974). 
17. Hsu, M. H., and Sauer, J. R. Sodium, Potassium, Chloride and water balance in the feeding lone star tick, Amblyomma americanum (Linneaus) (Acarina: Ixodidae). Journal of the Kansas Entomological Society 47, 536-537 (1974).

18. Kim, D., Maldonado-Ruiz, P., Zurek, L. \& Park, Y. Water absorption through salivary gland type I acini in the blacklegged tick, Ixodes scapularis. PeerJ 5, e3984 (2017).

19. Maldonado-Ruiz, L. P., Park, Y. \& Zurek, L. Liquid water intake of the lone star tick, Amblyomma americanum: Implications for tick survival and management. Scientific reports 10, 6000 (2020).

20. Sonenshine, D. E. Biology of Ticks. (Oxford University Press, 1991a).

21. Kim, D., Simo, L., Vancova, M., Urban, J. \& Park, Y. Neural and endocrine regulation of osmoregulatory organs in tick: Recent discoveries and implications. Gen Comp Endocrino/ 278, 42-49, doi:10.1016/j.ygcen.2018.08.004 (2019).

22. Nuttall, P. A. Wonders of tick saliva. Ticks and Tick-borne Diseases 10, 470-481, doi:https://doi.org/10.1016/j.ttbdis.2018.11.005 (2019).

23. Yoder J.A., B. J. B., Bundy M.R., Hedges B. Z. and Gribbins K.M. . Functional morphology of secretion by the large wax glands (Sensilla sagittiformia) Involved in tick defense. Psyche: A Journal of Entomology vol. 2009, 1-8, doi:doi.org/10.1155/2009/631030 (2009).

24. Walker, A. R., Lloyd, C., Mcguire, K., Harrison, S. J. \& Hamilton, J. Integumental glands of the tick Rhipicephalus appendiculatus (Acari:Ixodidae) as potential producers of semiochemicals. Journal of medical entomology 33 5, 743-759 (1996).

25. Pavis, C., Mauleon H Fau - Barre, N., Barre N Fau - Maibeche, M. \& Maibeche, M. Dermal gland secretions of tropical bont tick,Amblyomma variegatum (Acarina: Ixodidae): Biological activity on predators and pathogens. J.Chem.Ecol 7 (1994).

26. Hackman, R. H. \& Filshie, B. K. The tick cuticle. 1 (Pergamon Press, 1982).

27. Yoder, J. A., Pollack, R. J., Spielman, A., Sonenshine, D. E. \& Johnstons, D. E. Secretion of squalene by ticks. Journal of Insect Physiology 39, 291-296, doi:https://doi.org/10.1016/0022-1910(93)90059-Z (1993).

28. Diehl, P. A., Guerin P Fau - Vlimant, M., Vlimant M Fau - Steullet, P. \& Steullet, P. Biosynthesis, production site, and emission rates of aggregation-attachment pheromone in males of two Amblyomma ticks. J Chem Ecol 17, 833-847, doi:10.1007/BF01395594 (1991).

29. Lusby, W. R., Sonenshine, D. E., Yunker, C. E., Norval, R. A. \& Burridge, M. J. Comparison of known and suspected pheromonal constituents in males of the African ticks, Amblyomma hebraeum Koch and Amblyomma variegatum (Fabricius). Experimental \& Applied Acarology 13, 143-152, doi:10.1007/BF01193665 (1991).

30. Yoder, J. A., Hedges, B. Z., Tank, J. L. \& Benoit, J. B. Dermal gland secretion improves the heat tolerance of the brown dog tick, Rhipicephalus sanguineus, allowing for their prolonged exposure to host body temperature. Journal of Thermal Biology 34, 256-265, doi:https://doi.org/10.1016/j.jtherbio.2009.03.004 (2009). 
31. Folk, G. E. \& Semken, A. The evolution of sweat glands. International Journal of Biometeorology 35, 180-186, doi:10.1007/BF01049065 (1991).

32. Yoder, J. A., Benoit, J. B., Bundy, M. R., Hedges, B. Z. \& Gribbins, K. M. Functional Morphology of Secretion by the Large Wax Glands (Sensilla Sagittiformia) Involved in Tick Defense. Psyche 2009, 631030, doi:10.1155/2009/631030 (2009).

33. Walker, A. R., Lloyd Cm Fau - McGuire, K., McGuire K Fau - Harrison, S. J., Harrison Sj Fau - Hamilton, J. G. \& Hamilton, J. G. Integumental glands of the tick Rhipicephalus appendiculatus (Acari:Ixodidae) as potential producers of semiochemicals. J Med Entomo/ 33, 743-759 (1996).

34. Lees, A. D. Transpiration and the Structure of the Epicuticle in Ticks. The Journal of Experimental Biology 23, 379 (1947).

35. Walker, A. R., Lloyd, C. M., McGuire, K., Harrison, S. J. \& Hamilton, J. G. C. Integument and Sensillum Auriforme of the Opisthosoma of Rhipicephalus appendiculatus (Acari: Ixodidae). Journal of Medical Entomology 33, 734-742, doi:10.1093/jmedent/33.5.734 (2014).

36. Klompen, J. S., Keirans, J. E., Filippova, N. \& Oliver, J.

37. Clifford, C. M. \& Anastos, G. The Use of Chaetotaxy in the Identification of Larval Ticks (Acarina: Ixodidae). The Journal of Parasitology 46, 567-578, doi:10.2307/3274939 (1960).

38. Šimo, L. \& Park, Y. Neuropeptidergic control of the hindgut in the black-legged tick Ixodes scapularis. International Journal for Parasitology 44, 819-826, doi:https://doi.org/10.1016/j.ijpara.2014.06.007 (2014).

39. Karim, S., Kenny, B., Troiano, E. \& Mather, T. N. RNAi-mediated gene silencing in tick synganglia: a proof of concept study. BMC biotechnology 8, 30-30, doi:10.1186/1472-6750-8-30 (2008).

40. Schneider, C. A., Rasband, W. S. \& Eliceiri, K. W. NIH Image to ImageJ: 25 years of image analysis. Nature Methods 9, 671-675, doi:10.1038/nmeth.2089 (2012).

41. Simo, L., Zitnan, D. \& Park, Y. Two novel neuropeptides in innervation of the salivary glands of the black-legged tick, Ixodes scapularis: myoinhibitory peptide and SIFamide. The Journal of comparative neurology 517, 551-563, doi:10.1002/cne.22182 (2009).

42. Simo, L., Koči, J. \& Park, Y. Receptors for the neuropeptides, myoinhibitory peptide and SIFamide, in control of the salivary glands of the blacklegged tick Ixodes scapularis. Insect biochemistry and molecular biology 43, 376-387, doi:10.1016/j.ibmb.2013.01.002 (2013).

43. Kim, D., Šimo, L. \& Park, Y. Molecular characterization of neuropeptide elevenin and two elevenin receptors, IsElevR1 and IsElevR2, from the blacklegged tick, Ixodes scapularis. Insect Biochemistry and Molecular Biology 101, 66-75, doi:https://doi.org/10.1016/j.ibmb.2018.07.005 (2018).

44. Kim, D., Urban, J., Boyle, D. L. \& Park, Y. Multiple functions of Na/K-ATPase in dopamine-induced salivation of the Blacklegged tick, Ixodes scapularis. Sci Rep 6, 21047, doi:10.1038/srep21047 (2016).

45. Jan, L. Y. \& Jan, Y. N. Antibodies to horseradish peroxidase as specific neuronal markers in Drosophila and in grasshopper embryos. Proc Natl Acad Sci U S A 79, 2700-2704, doi:10.1073/pnas.79.8.2700 (1982). 
46. Seppo, A., Matani P Fau - Sharrow, M., Sharrow M Fau - Tiemeyer, M. \& Tiemeyer, M. Induction of neuron-specific glycosylation by Tollo/Toll-8, a Drosophila Toll-like receptor expressed in non-neural cells. Development 130, 1439-1448, doi:10.1242/dev.00347 (2003).

\section{Tables}

Table 1. Compounds injected into ticks for dermal secretion responses. Further details of the compound information are in the materials and methods.

\begin{tabular}{|clll|}
\hline Compound & Concentration & N & Response \% \\
\hline Biogenic amines & & & \\
\hline Octopamine & $10 \mathrm{mM}$ & 10 & $0 \%$ \\
\hline Norepinephrine & $10 \mathrm{mM}$ & 10 & $0 \%$ \\
\hline Dopamine & $10 \mathrm{mM}$ & 10 & $10 \%$ \\
\hline Serotonin & $1 \mathrm{mM}$ & 20 & $75 \%$ \\
\hline Neuropeptides & & & \\
\hline SIFamide & $10 \mathrm{mM}$ & 10 & $0 \%$ \\
\hline MIP-1 & $1 \mathrm{mM}$ & 10 & $0 \%$ \\
\hline Proctolin & $10 \mathrm{mM}$ & 5 & $0 \%$ \\
\hline ELV-1 & $10 \mathrm{mM}$ & 10 & $10 \%$ \\
\hline Secondary signaling messengers & & & \\
\hline Dibutyryl cAMP & $10 \mathrm{mM}$ & 5 & $0 \%$ \\
\hline Forskolin & $10 \mathrm{mM}$ & 10 & $10 \%$ \\
\hline Dibutyryl cGMP & $10 \mathrm{mM}$ & 20 & $10 \%$ \\
\hline SNAP & $5 \mathrm{mM}$ & 10 & $20 \%$ \\
\hline
\end{tabular}

\section{Figures}



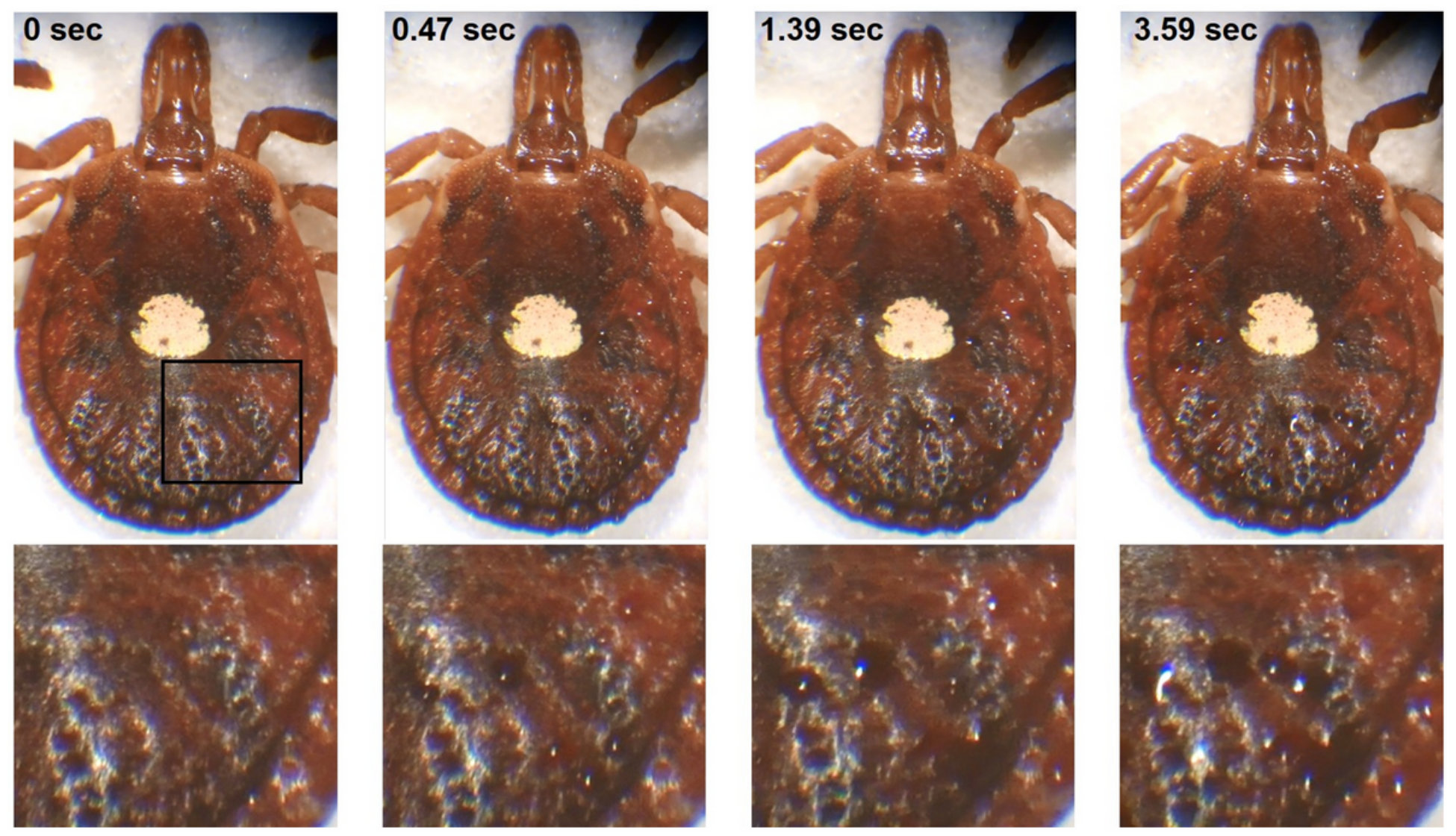

\section{Figure 1}

Dermal secretion upon heat probe contact. Time 0 represents the moment the heated probe contacted the legs of the ticks. Top images show the full dorsal view of the tick over the course of 4 seconds after touching the dorsum with the heated probe. Lower images show the magnification of the insertion in the time 0 for each time point. Real time secretory response can be observed in supplementary Video S1.

a

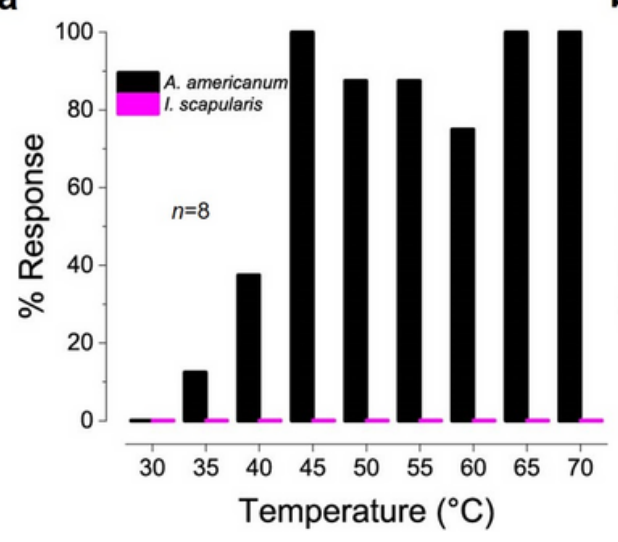

b

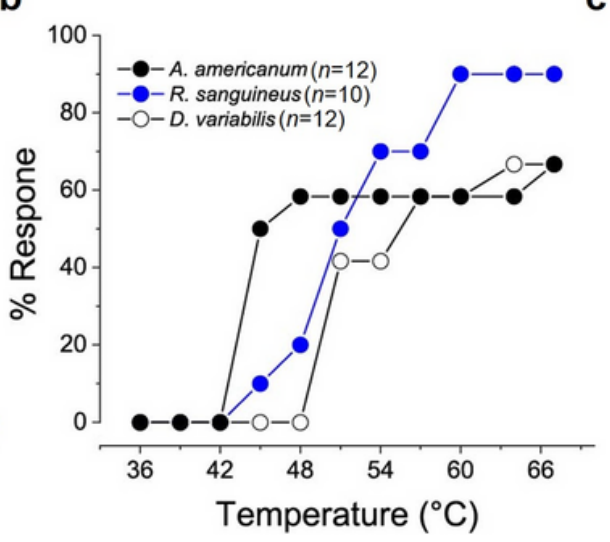

C

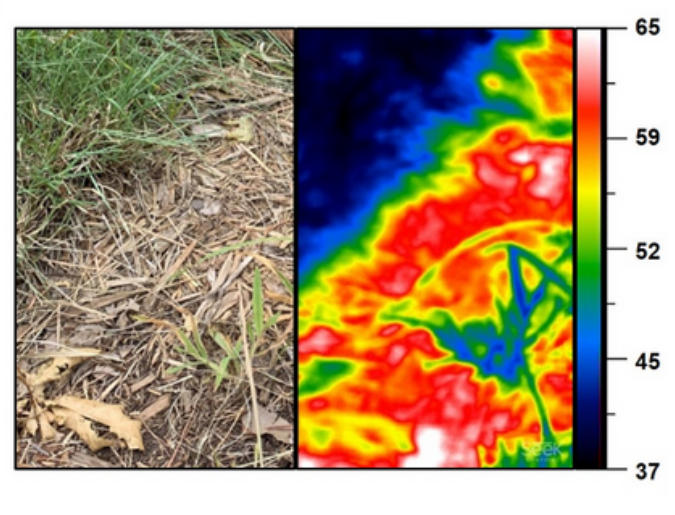

Figure 2

Dermal secretion in response to heat probe contact. a) The response by percentage for dermal secretion at different temperature in female Amblyomma americanum ( $n=8$ ticks/temperature). b) Dermal secretion responses to a heat probe contact tested in a same tick with sequentially increased temperature of the heat probe. c) Thermal image with regular bright field image for a typical tick habitat showing the 
temperature range of 38 to $650 \mathrm{C}$. The image was captured in a sunny summer day with $33 \mathrm{oC}$ ambient temperature.

a

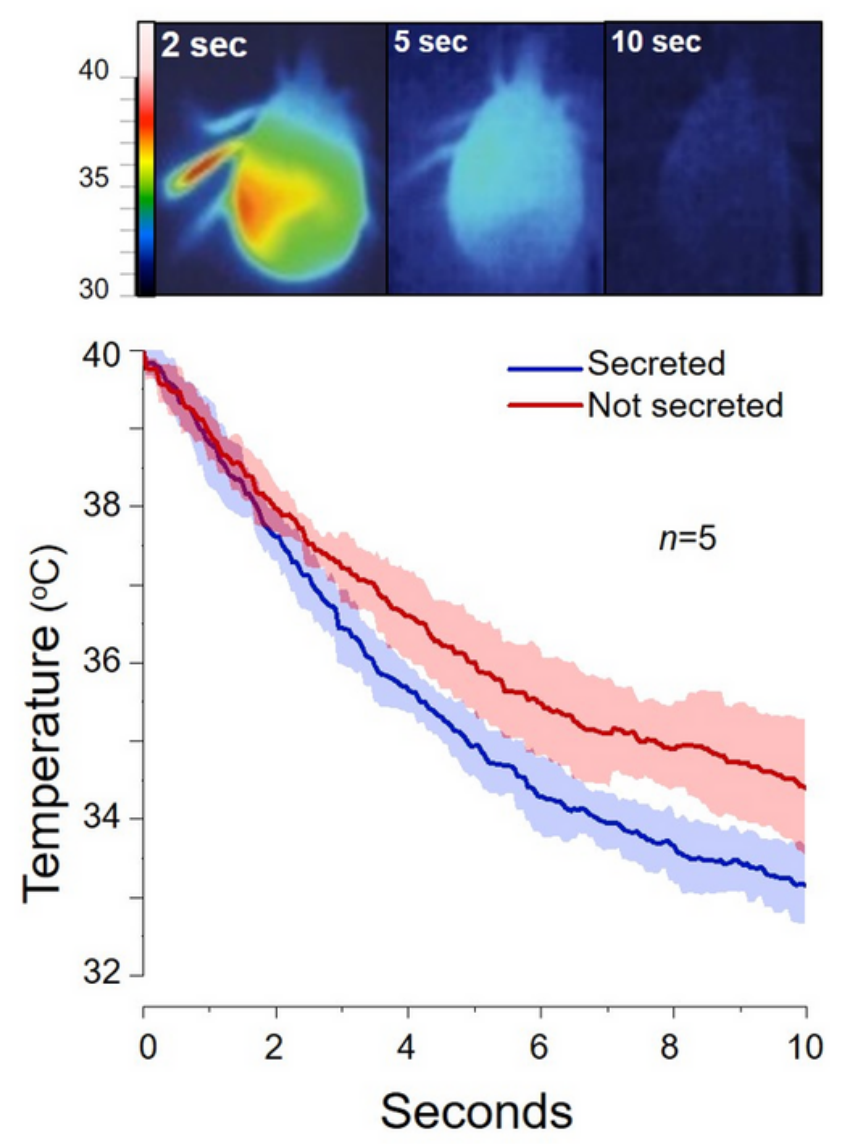

b
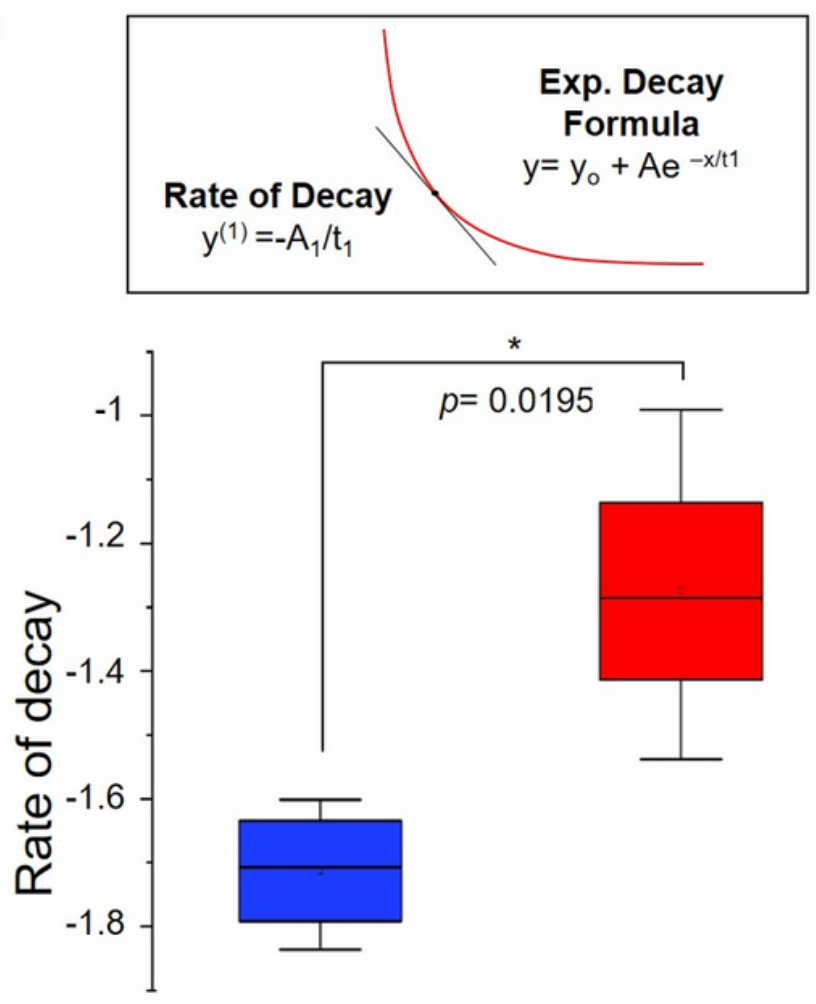

Secreted

Not secreted

\section{Figure 3}

Cooling rate with and without dermal secretion after the contact with heat probe. a) Cooling rate measured for 10 seconds after contact with heat probe in Amblyomma americanum. The blue line represents the ticks with dermal secretion and the red line is for the ticks without secretion $(n=5$ ticks/treatment). Shadowed area represents the SEM for each curve. b) The regression formula and lines for the rate of decay that was used for the statistics by Student's t-test. Box plots show the median, 1st and 3rd quartile for the box and 95 and $5 \%$ for the error bars. 


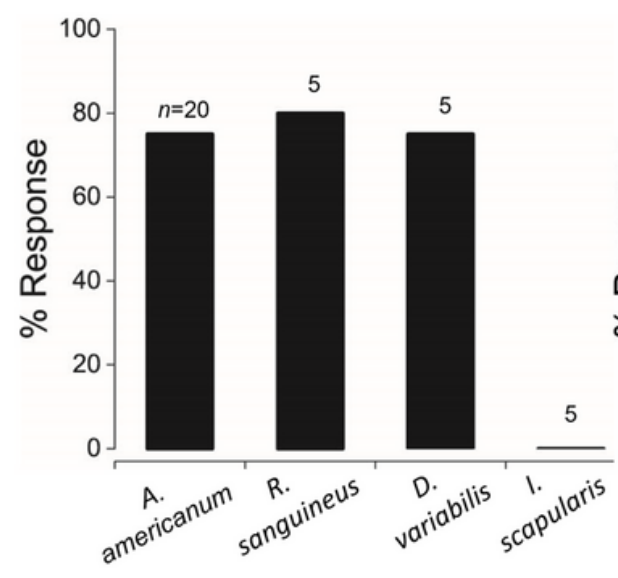

Serotonin $1 \mathrm{mM}(10 \mathrm{nl})$

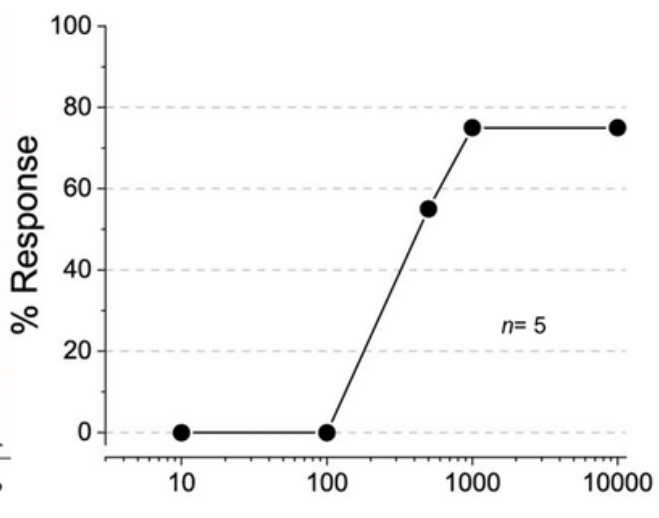

$\log (\mathrm{nM}[$ serotonin])

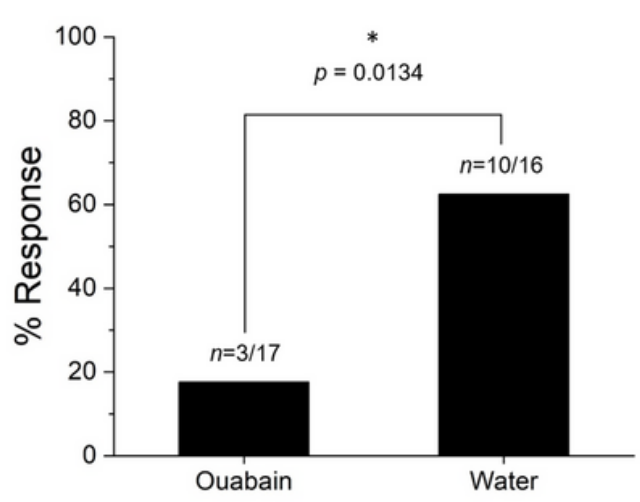

Serotonin 1mM (10 nl)

\section{Figure 4}

Dermal secretion induced by serotonin injection. a) The dermal secretion responses by percentage after serotonin injection (10 $\mathrm{nl}$ of $1 \mathrm{mM}$ serotonin) in Metastriata ticks, Amblyomma americanum, Rhipicephalus sanguineus, Dermacentor variabilis, and Ixodes scapularis. Supplementary Video S2 shows the real time response upon serotonin injection. b) Dose-response to serotonin injection in A. americanum (10 $\mathrm{nl}$ injections). c) Effect of ouabain pre-treatment on the serotonin-mediated dermal secretion. Ouabain pre-treatment was made with an injection of $10 \mathrm{nl}$ of $100 \mu \mathrm{M}$ at 30 minutes before the serotonin treatment. Water injections were used for the control. The statistics were Chi-square test.
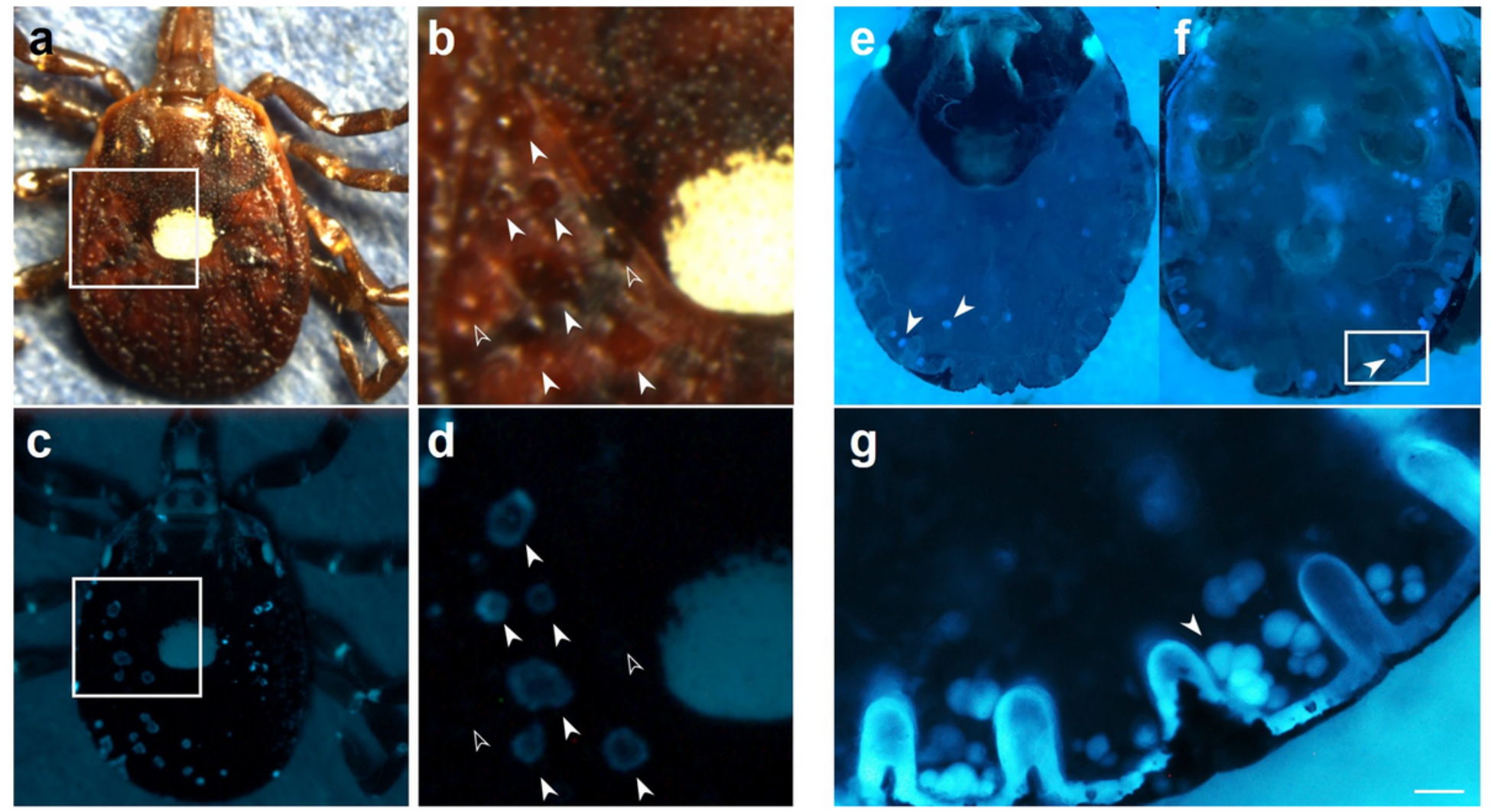

Figure 5 
Localization of dermal secretion and putative type II gland. a) Dorsal view of Amblyomma americanum female after dermal secretion stimulated by heat exposure on the legs. The white square in $A$ and $C$ highlights a subset of secretion spots magnified in B and D. b) Magnified view of the white box in A, where white arrowheads point the dermal secretions dried on the top of the pores leaving fluorescent residue and the empty arrow heads point to secretions which are not showing blue fluorescence. c) Image A under CFP filter set. d) Magnified view of the white box in C, where white arrowheads the dried drops of secretion with fluorescent residue and the empty arrow heads point to secretions with no blue fluorescence. e) Internal view of dorsal integument with CFP filter set and f) Internal view of ventral integument. White arrowheads point to the putative dermal glands type II. g) Magnified view of the inset shown in $\mathrm{F}$ where white arrow heads point to a cluster of type II glands located on the ticks festoons. Scale bar shown at $100 \mu \mathrm{m}$. 


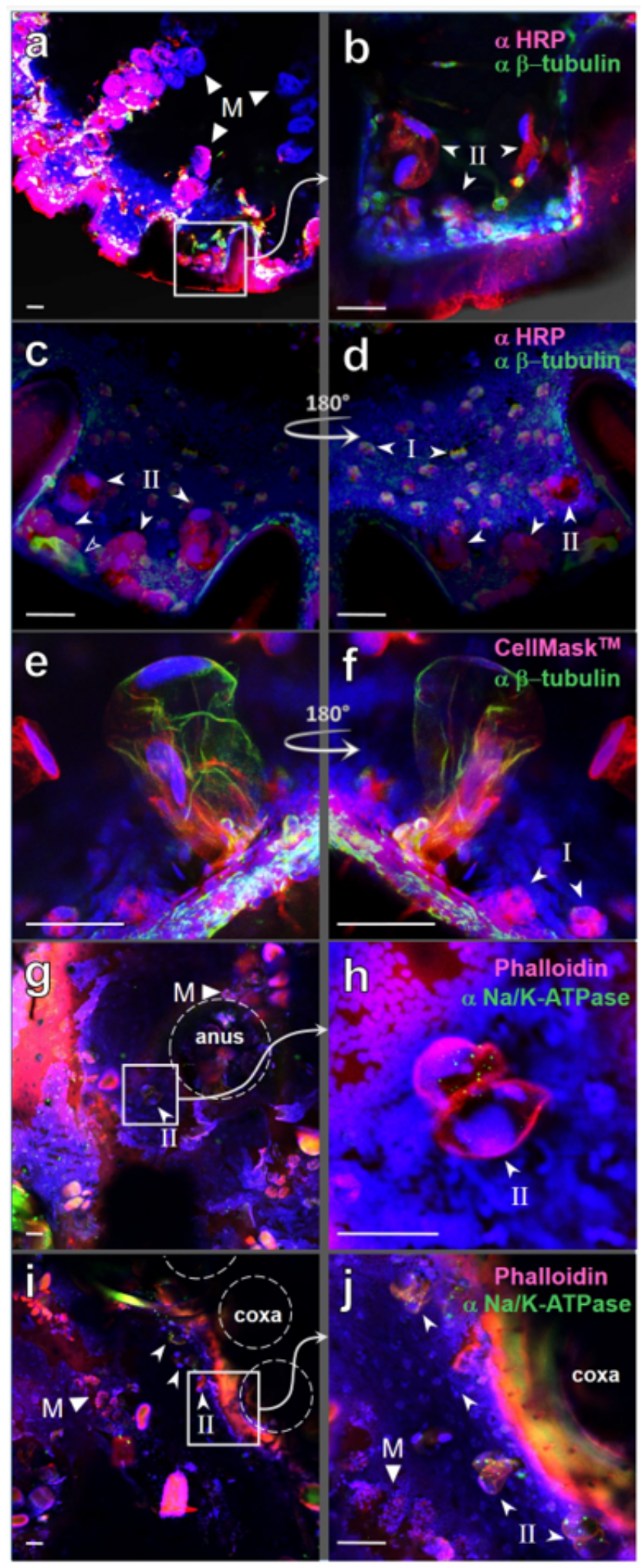

\section{Figure 6}

Confocal images of the dermal layer showing type II and type I glands. a) Ventral layer of unfed tick. The white triangles points to the clusters of round shapes are for the dorso-ventral muscle (M). The inset containing red (anti-HRP) is a festoon showing typical type II glands magnified in B. b) Inset of A showing the type II dermal glands indicated by white arrowheads. c) A confocal image with a Z-stack for 9 layers of a total of $27.54 \mu \mathrm{m}$. The white arrowheads point to type II dermal glands, empty arrowhead points the 
type II gland stained for $\beta$-tubulin. d) Lower layers of the region shown in C, a Z-stack for 12 layers of a total of $36.72 \mu \mathrm{m}$. This image shows both type I type II glands. The front and back views were made by different degrees of transparency in the Z-stack algorism. e) A paired type II gland with the nuclei staining in each. The image is the Z-stack of 14 layers of total $37.38 \mu \mathrm{m}$. f) The backside view of E made by different degrees of transparency in the Z-stack. Note that $\beta$-tubulin immunoreactivity is clustered in the basal region of the gland. Type I glands are marked with the arrowheads. g) Type II glands in the central/anus region of the ventral dermal layer, where white square shows type II glands and white triangle the dorso-ventral muscles (M). h) Inset of G showing the type II dermal glands. Note spotted granular green staining of $\mathrm{Na} / \mathrm{K}$-ATPase. i) Type II glands in the lateral/coxa region of the ventral dermal integument, where white arrowheads are for type II glands and white triangle the dorso-ventral muscles (M). j) Inset of I showing the type II dermal glands with white arrowheads and spotted granular staining of $\mathrm{Na}$ /K-ATPase. White triangle is for the dorso-ventral muscles (M). a-d: Blue; nuclei staining with Hoeschst, Green; $\beta$-tubulin staining and Red; Horseradish Peroxidase staining (CyTM3). e and f: nuclei staining with Hoeschst, Green; $\beta$-tubulin and Red; plasma membrane stained with CellMaskTM. g-j: nuclei staining with Hoeschst, Green; Na/K-ATPase and Red; Phalloidin used for actin staining. All scale bars shown represent $50 \mu \mathrm{m}$.
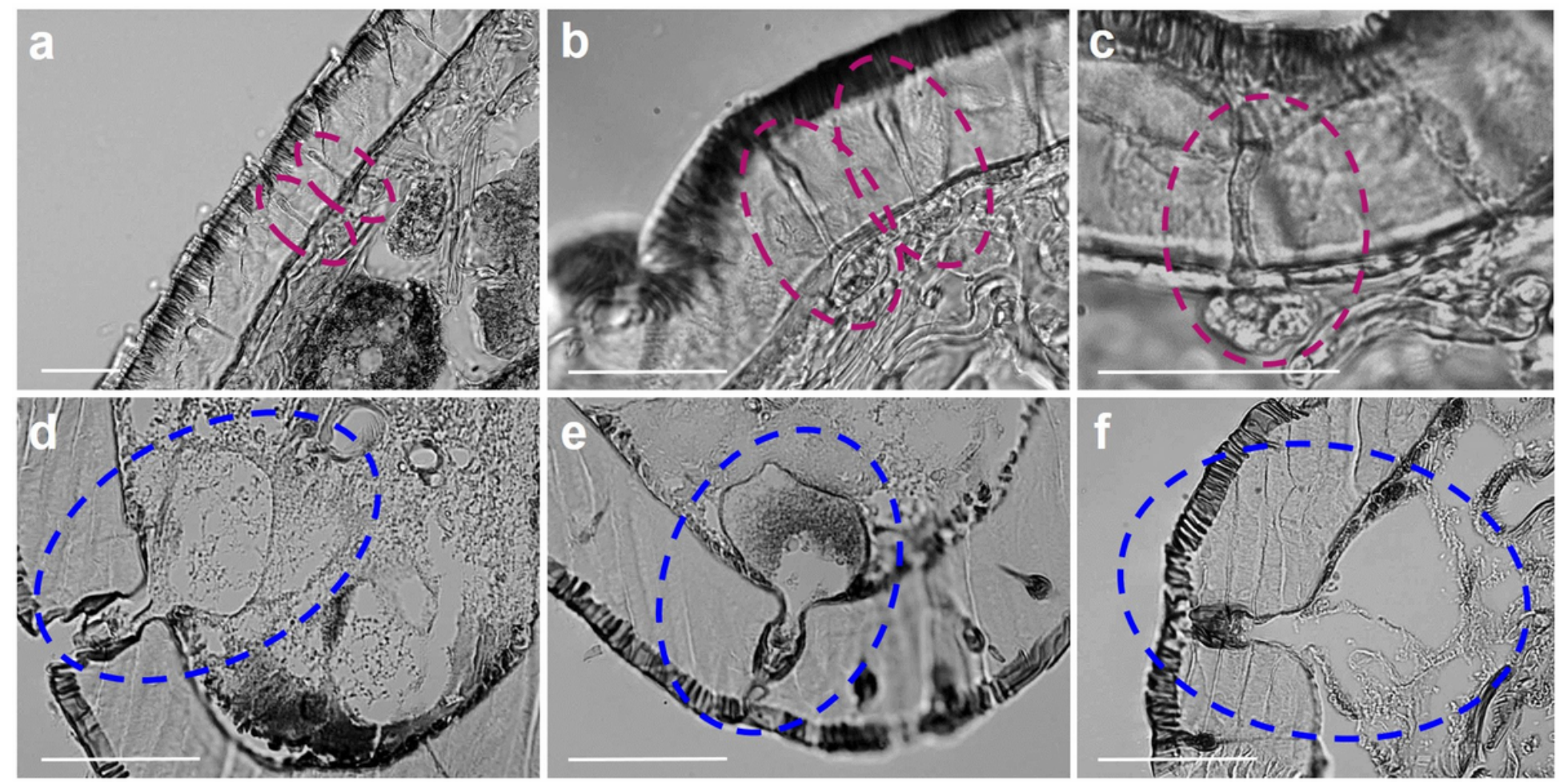

\section{Figure 7}

Dermal gland type I and II associated with the ducts on cuticle layer. Magenta circles (a, b, and c) show the pore associated with the dermal gland type I, blue circles (D, E, and F) show wide pores associated with the dermal gland type II. a) Unfed tick integument showing type I dermal glands of an unfed female tick. b and c) Close-up image of type I glands characterized by thin pore and small cell clusters. $d-f$ ) Type II glands shown in different locations of alloscutum of unfed ticks. A part of glandular structures are connected to the wide ducts on the cuticular layer. Scale bar is for $100 \mu \mathrm{m}$. 


\section{Supplementary Files}

This is a list of supplementary files associated with this preprint. Click to download.

- Supplementary.pdf

- VideoS1.mp4

- VideoS2.mp4 